EXTENDED REPORT

\title{
Arthritis instantaneously causes collagen type I and type II degradation in patients with early rheumatoid arthritis: a longitudinal analysis
}

\author{
R B M Landewé, P Geusens, D M F M van der Heijde, M Boers, S J van der Linden, \\ P Garnero
}

Ann Rheum Dis 2006;65:40-44. doi: 10.1136/ard.2004.035196

See end of article for authors' affiliations

............

Correspondence to: Dr Robert Landewé, University Hospital Maastricht, PO Box 5800 , 6202AZ Maastricht Netherlands; RLAN@SINT. AZM.NL

Accepted 29 May 2005 Published Online First 26 August 2005
Background: Markers of collagen type I (CTX-1) and type II (CTX-II) degradation, reflecting bone and cartilage breakdown, appear to predict long term radiographic progression in chronic persistent arthritis. Objective: To analyse longitudinally whether changes in arthritis severity are linked to immediate changes in the level of CTX-I and CTX-II degradation.

Methods: CTX-I and CTX-II were measured in urine samples from 105 patients with early rheumatoid arthritis who had participated in the COBRA trial at baseline and at 3,6,9, and 12 months after the start of treatment. The course of the biomarkers over time was compared with the course of ESR, swollen and tender joint counts, and 28 joint disease activity score (DAS28), measured at the same time points, with adjustment for rheumatoid factor, treatment, and baseline radiographic damage, by generalised estimating equations (GEE) with first order autoregression.

Results: GEE showed that CTX-I was longitudinally associated with DAS28, but not with ESR, swollen joint count, or tender joint count. CTX-II, however, was longitudinally associated with ESR, swollen joint count and DAS28, but not with tender joint count. The longitudinal association implies that an increase in the extent of arthritis is immediately followed by an increase in collagen type II degradation, and to a lesser extent collagen type I degradation.

Conclusions: Cartilage degradation as measured by CTX-II and to a lesser extent bone degradation as measured by CTX-I closely follows indices of arthritis. Clinically perceptible arthritis is responsible for immediate damage, which will become visible on plain $x$ rays only much later.
J

oint damage in rheumatoid arthritis is considered to be the result of chronic inflammation, as demonstrated in several studies. ${ }^{1-4}$ It is not known whether arthritis has an immediate influence on the bone and cartilage in joints.

Technically, a comparative analysis between the extent of arthritis and the occurrence of radiographic damage is hampered by the fact that measures of arthritis behave like process variables with a high rate of fluctuation, whereas radiographic damage slowly cumulates over time without important fluctuations in individual patients. Recently, we showed that both collagen type I degradation products (CTXI) and, especially, collagen type II degradation products (CTX-II), measured in the urine of patients participating in the COBRA study (Combinatietherapie Bij Reumatoide Artritis) at baseline, were highly predictive of five year radiographic progression, especially in patients without visible baseline damage. ${ }^{5}$ In a subsequent analysis we showed that early changes of CTX-II induced by therapy were predictive of radiographic progression independent of markers of disease activity. ${ }^{6}$ As collagen type II degradation may reflect cartilage destruction in the joints of patients with rheumatoid arthritis, ${ }^{7}$ CTX-II is potentially a useful and specific biomarker for forthcoming radiographic progression, and may be used for the follow up of treatment in early rheumatoid arthritis. Because CTX-I and CTX-II behave like process variables, these biomarkers are potentially more appropriate to investigate the relation between inflammation and bone and cartilage destruction than radiographic damage.

In this study we addressed the hypothesis that the presence and extent of arthritis has immediate repercussions for bone and cartilage degradation, by analysing the longitudinal relation between markers of arthritis and collagen degradation products, on the assumption that the latter reflect forthcoming structural damage.

\section{METHODS \\ Patients}

We investigated patients participating in the COBRA study. COBRA was a 56 week multicentre clinical trial that randomly assigned 155 patients who met the American College of Rheumatology criteria for rheumatoid arthritis. ${ }^{8}{ }^{9}$ All patients had early active disease (less than two years, median four months). None of the patients had been treated with disease modifying anti-rheumatic drugs (DMARDs) previously. One group was treated with a combination of sulfasalazine, methotrexate, and, initially, high dose oral prednisolone (COBRA regimen); the other group was treated with sulfasalazine alone. The prednisolone dose was $60 \mathrm{mg} /$ day in the first two weeks and tapered in weekly steps to the maintenance dosage of $7.5 \mathrm{mg} /$ day in week 7. Prednisolone and methotrexate were tapered and stopped after weeks 28 and 40, respectively, while sulfasalazine was continued.

Non-fasting second morning void urine samples were obtained at baseline, three months, six months, nine months, and at the end of the trial, and were kept frozen at $-20^{\circ} \mathrm{C}$.

The current report is based on the 110 patients who had urine available at both baseline and at least one follow up

Abbreviations: COBRA, Combinatietherapie Bii Reumatoide Artritis trial; CTX-I, collagen type I degradation products; CTX-II, collagen type II degradation products; GEE, generalised estimating equations 


\begin{tabular}{|lll|}
\hline Table 1 Baseline characteristics \\
\hline & $\begin{array}{l}\text { Sulfasalazine } \\
\text { monotherapy } \\
(\mathbf{n}=58)\end{array}$ & $\begin{array}{l}\text { COBRA therapy } \\
(\mathbf{n}=52)\end{array}$ \\
\hline $\begin{array}{lll}\text { Age (years) (mean (SD)) } \\
\text { Disease duration (months) }\end{array}$ & $49(12)$ & $50(13)$ \\
(mean (SD)) & $5(5)$ & $5(5)$ \\
Female (\%) & 52 & 71 \\
RF positive (\%) & 72 & 74 \\
Erosive disease (\%) & 44 & 42 \\
SE (nil/heterozygous/ & $47 / 38 / 15$ & $46 / 48 / 6$ \\
homozygous) (\%) & \\
\hline $\begin{array}{l}\text { COBRA, Combinatietherapie Bii Reumatoide Artritis trial; RF, rheumatoid } \\
\text { factor; SE, shared epitope. }\end{array}$ \\
\hline
\end{tabular}

visit, comprising 52 COBRA treated and 58 sulfasalazine treated patients. During and after the trial, swollen and tender joint counts were carried out, as well as an assessment of global wellbeing (visual analogue scale) and the Westergren erythrocyte sedimentation rate (ESR). Disease activity was expressed as the 28 joint disease activity score (DAS28), a validated index composed of a 28 joint swelling and tenderness count, an acute phase reactant (ESR), and a measure of global wellbeing ( $10 \mathrm{~cm}$ visual analogue scale).

Urinary C-terminal cross linking telopeptide of type-I collagen (CTX-I) was measured by the Crosslaps enzyme linked immunosorbent assay (ELISA) (Nordic Biosciences, Herlev, Denmark). This assay uses a polyclonal antiserum raised against $\beta$-isomerised EKAH $\beta$ DGGR sequence of the C-telopeptide of $\alpha \mathrm{l}$ chains of human type I collagen. Intraassay and interassay coefficients of variation are less than $6 \%$ and $9 \%$, respectively.

Urinary C-terminal cross linking telopeptide of type II collagen (CTX-II) was measured by a competitive ELISA (Cartilaps, Nordic Biosciences) based on a mouse monoclonal antibody raised against the EKGPDP sequence of human type-II collagen C-telopeptide. This sequence, in which the lysine residue $(\mathrm{K})$ participates in cross linking between type II collagen molecules, is present in the mature articular collagen network produced by chondrocytes. Thus detection of this sequence in urine is considered to specifically reflect the degradation of mature type-II collagen molecules.

Intra-assay and interassay coefficients of variation are less than $8 \%$ and $10 \%$, respectively. The average intraindividual coefficient of variation over 24 hours, reflecting diurnal variability, was only $15 \%$.

Urinary CTX-I and CTX-II levels were corrected by the urinary creatinine $(\mathrm{Cr})$ concentration measured by a standard colorimetric method. All measurements of CTX-I and CTX-II were carried out in a central laboratory (Synarc, Lyon, France).

None of the patients suffered from marked liver or kidney function impairment that might have affected the urinary levels of CTX-I or CTX-II.

\section{Statistical analysis}

CTX-I and CTX-II levels were logarithmically transformed, as both variables had a skewed distribution at all time points.
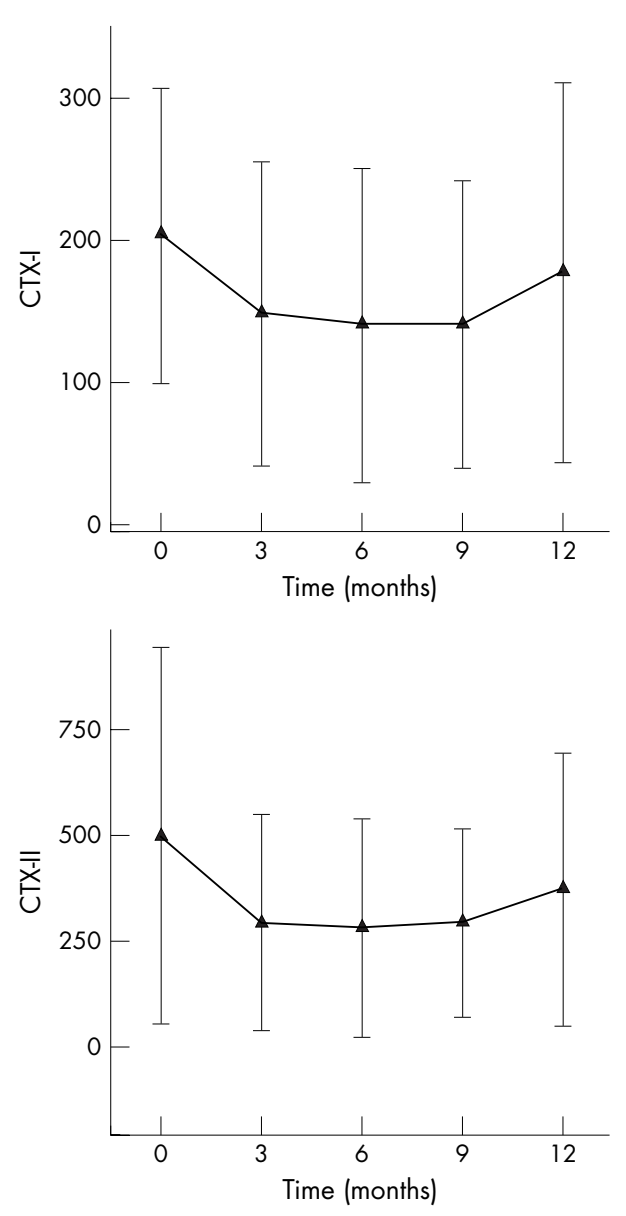

Figure 1 Course of collagen type I and type II degradation products in patients with rheumatoid arthritis who have participated in the COBRA study. Symbols are means; error bars $=\mathrm{SD}$.

\section{Longitudinal data analysis}

Longitudinal datasets are characterised by observations with a high variability between patients and a rather low variability within patients. The high within-patient correlation means that longitudinal relations cannot be analysed using ordinary regression methods. GEE (generalised estimating equations) is a regression technique to study intervariable relations in longitudinal studies; it takes time, as well as time independent and time dependent covariates into account. ${ }^{10}$ The advantages of GEE over ordinary methods are that GEE uses all available longitudinal data, allows unequal numbers of repeated measurements and unequal time intervals, and does not require multivariate normality of the outcome variable. GEE does require an a priori "working" correlation structure in order to adjust for the within-subject correlation operating in repeated measurement designs. A correlation structure must be chosen on the basis of the actual dataset. In this study the "exchangeable" correlation structure was appropriate for all outcome measures, because in the correlation matrix all correlations at different time

Table 2 Number of assessments per time point

\begin{tabular}{llcclll}
\hline & Baseline & 3 Months & $\mathbf{6}$ Months & $\mathbf{9}$ Months & $\mathbf{1 2}$ Months & Total \\
\hline CTX-I & 111 & 98 & 96 & 81 & 84 & 470 \\
CTX-II & 114 & 105 & 101 & 88 & 87 & 495 \\
\hline
\end{tabular}

Figures represent numbers of valid assessments. 
Table 3 Modelling biomarkers of collagen type I and type II degradation in time

\begin{tabular}{lll}
\hline & \multicolumn{2}{l}{ Regression coefficient } \\
\cline { 2 - 3 } & $\ln$ CTX-I & $\ln$ CTX-II \\
\hline Constant & 5.2 & 5.9 \\
Time & $-0.17(\mathrm{p}<0.001)$ & $-0.18(\mathrm{p}<0.001)$ \\
Time $^{2}$ & $1.8(\mathrm{p}<0.001)$ & $2.1(\mathrm{p}<0.001)$ \\
Time $^{3}$ & $\mathrm{NS}$ & $\mathrm{NS}$ \\
\hline CTX-I, collagen type I; CTX-II, collagen type II. \\
\hline
\end{tabular}

points were approximately equal (Spearman correlation coefficients for CTX-I and CTX-II differed between 0.45 and $0.60)$.

\section{Model building}

The effect of time on CTX-I and CTX-II

We first tried to describe the course of CTX-I and CTX-II in time mathematically, by investigating the relation between CTX-I/CTX-II and time, and subsequently adding time ${ }^{2}$ and time $^{3}$ in order to increase the fit of the model (model 1).

\section{Baseline factors (time independent)}

We subsequently investigated whether variables known to be predictive of radiographic outcome (treatment, rheumatoid factor status, baseline damage) significantly contributed to explaining variance (model 2).

\section{Longitudinal factors (time dependent)}

We then added variables reflecting the extent of clinically perceptible arthritis to model 3, in order to investigate the longitudinal relation between arthritis and CTX-I/CTX-II (model 3). This model was run several times, every time with a different variable reflecting arthritis.

\section{Autoregressive analysis}

We then added to model 3 the levels of CTX-I/CTX-II that were measured one time point earlier (three months) (CTX$\mathrm{I}_{\mathrm{t}-3}$ months, CTX-II $\mathrm{I}_{\mathrm{t}-3}$ months $)$ in order to investigate whether the relations found in model 3 justified a longitudinal interpretation.

\section{Time lag analysis}

This analysis was run to investigate the temporal relation between arthritis and CTX-I/CTX-II. In model 4, the concurrent assessments of arthritis were substituted by assessments of arthritis three months or six months earlier. The correlations between variables of arthritis (ESR, swollen joint count, tender joint count, DAS28) and CTX-I or CTX-II were compared by using standardised regression coefficients. A standardised regression coefficient describes the relation between a standardised (normalised) independent variable (here, the variables reflecting arthritis) and the dependent variable (here, CTX-I or CTX-II), and can be used to weight correlations between different independent variables and one dependent variable.

\section{RESULTS}

Table 1 summarises the patients included in the study. All patients had active rheumatoid arthritis of very short duration; many had predictors of unfavourable prognosis, such as rheumatoid factor, erosions at baseline, and the HLADR4 genotype. None of the patients had used DMARDs previously.

Table 2 summarises the number of observations on which this analysis was based. The number of assessments was somewhat greater in the early part of the study than in the later part for both CTX-I and CTX-II. A complete profile (five measurements) could be obtained for 64 patients for CTX-I, and for 72 patients for CTX-II. A near complete profile (at least four of five measurements) could be obtained in 82 patients for CTX-I and for 94 patients for CTX-II.

\section{Time course}

The course of CTX-I and CTX-II over time is shown in fig 1. Curve fitting (GEE model 1) showed that a quadratic function of time fitted the data best for both CTX-I and CTX-II (table 3).

\section{Baseline factors}

Subsequently, we added to the models described above the three baseline factors which we knew were predictors of radiographic progression. None of these factors significantly contributed to explaining the variance in CTX-I. Treatment allocation and rheumatoid factor status were independent baseline predictors in the CTX-II model: COBRA was associated with significantly lower values of CTX-II than sulfasalazine; RF positivity was associated with significantly higher levels of CTX-II than RF negativity. We decided to enter all three baseline factors in all subsequent models, irrespective of their contribution.

\section{Longitudinal analysis}

Subsequently, several variables reflecting the extent of inflammation (arthritis), assessed at the same time points as CTX-I and CTX-II, were added to the model, which already involved time variables (time and time $^{2}$ ) and baseline variables (RF, treatment allocation, and baseline radiographic damage). In order to allow a longitudinal interpretation of the association between arthritis and CTX-I/CTX-II, the dependent variable CTX-I or CTX-II was further adjusted for the level of CTX-I/CTX-II at one time point of measurement earlier (first order autoregression), so that the change in CTX-I/CTX-II as compared with the previous measurement, rather than the absolute level of CTX-I/CTX-II, is the subject of investigation. An example of a longitudinal interpretation is given below.

Table 4 (A and $\mathrm{B}$ ) shows the results of this longitudinal analysis. The regression coefficients describe the relation between the assessment of arthritis extent and $\ln \mathrm{CTX}-\mathrm{I} /$ InCTX-II. The standardised regression coefficients allow a comparison between different types of assessment. Only the DAS28 was significantly longitudinally associated with InCTX-I. The separate components of DAS28 (ESR, swollen joint count, tender joint count) were not significantly associated. The interpretation of a longitudinal association between DAS28 and $\ln$ CTX-I is as follows: suppose an individual patient has a DAS28 of 5 , to which an $\ln$ CTX-I value of 4.2 is attached. If the DAS28 increases from 5 to 7 , the $\ln$ CTX-I level will instantaneously increase from 4.2 to $4.2+(7-5) \times 0.40=5.0$ (the regression coefficient for the relation between DAS28 and $\ln$ CTX-I is 0.40 ).

The relation between the extent of arthritis and $\ln$ CTX-II was much stronger than that between the extent of arthritis and $\operatorname{lnCTX}$-I (higher standardised regression coefficients), and involved the ESR, the swollen joint count, and the DAS28. The tender joint count was not significantly associated.

\section{Time lag analysis}

A time lag analysis was undertaken to explore further the longitudinal relation described here between markers of the extent of arthritis and markers of collagen degradation. Table 5 shows the consequences of the time lag analysis on the magnitude of the standardised regression coefficient. Time lags of three and six months between the marker of the extent of arthritis and CTX-II significantly decreased the level 
Table 4 Longitudinal regression analysis by generalised estimating equations for the relation between clinical variables of inflammation and biomarkers of joint destruction

\begin{tabular}{|c|c|c|c|c|}
\hline & $\begin{array}{l}\text { Regression } \\
\text { coefficient (RC) }\end{array}$ & $\begin{array}{l}\text { Standardised regression } \\
\text { coefficient }\end{array}$ & $\begin{array}{l}95 \% \text { confidence interval } \\
\text { of RC }\end{array}$ & $\mathrm{p}$ Value \\
\hline \multicolumn{5}{|c|}{ (A) Collagen type 1 degradation (CTX-1) } \\
\hline $\mathrm{ESR}(\mathrm{mm})$ & 0.012 & 0.05 & -0.01 to 0.026 & 0.072 \\
\hline Swollen joint count (0-28) & 0.037 & 0.04 & -0.019 to 0.093 & 0.20 \\
\hline Tender joint count $(0-28)$ & 0.032 & 0.03 & -0.018 to 0.081 & 0.22 \\
\hline DAS28 & 0.40 & 0.09 & 0.16 to 0.67 & 0.001 \\
\hline \multicolumn{5}{|c|}{ (B) Collagen type II degradation (CTX-II) } \\
\hline ESR $(\mathrm{mm})$ & 0.0079 & 0.24 & 0.005 to 0.01 & $<0.001$ \\
\hline Swollen joint count (0-28) & 0.021 & 0.17 & 0.011 to 0.031 & $<0.001$ \\
\hline Tender joint count (0-28) & 0.007 & 0.08 & -0.001 to 0.021 & 0.089 \\
\hline DAS28 & 0.11 & 0.22 & 0.066 to 0.15 & $<0.001$ \\
\hline \multicolumn{5}{|c|}{ 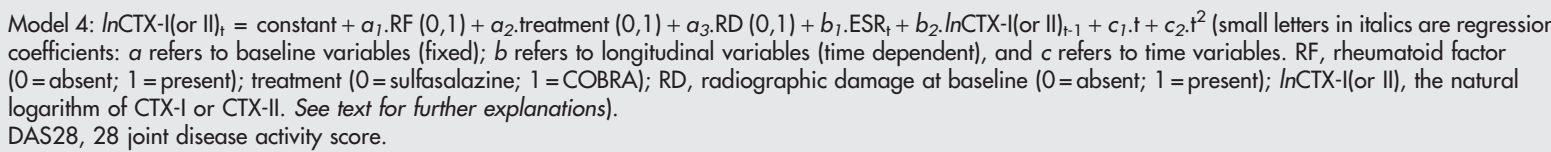 } \\
\hline
\end{tabular}

of association, as expressed by the standardised regression coefficients in table 5 . The relation was stronger for a time lag of three months than for one of six months.

Because the relation between markers of the extent of arthritis and CTX-I was much weaker than that between markers of the extent of arthritis and CTX-II, we present the time lag analysis only for CTX-II.

\section{DISCUSSION}

The analysis presented here proves there is a tight and direct longitudinal relation between clinically perceptible signs of arthritis, such as swollen joints and increased acute phase reactants, and degradation of collagen type I (CTX-I) and type II (CTX-II). The interpretation of the results of the analysis is that inflammation immediately causes collagen type I and type II degradation. Such a relation was already

Table 5 Longitudinal regression analysis by generalised estimating equations on the levels of CTX-II, with longitudinal variables representing arthritis added with a time lag of three or six months

\begin{tabular}{llll}
\hline & \multicolumn{2}{l}{ Standardised regression coefficient } \\
\cline { 2 - 4 } & No time lag & $\begin{array}{l}\text { Time lag } \\
\text { 3 months }\end{array}$ & $\begin{array}{l}\text { Time lag } \\
\text { 6 months }\end{array}$ \\
\hline Swollen joint count & $0.17^{* * *}$ & $0.14^{* *}$ & $0.11^{*}$ \\
Tender joint count & 0.08 & 0.02 & 0.00 \\
ESR & $0.24^{* * *}$ & $0.13^{*}$ & 0.10 \\
DAS28 & $0.22^{* * *}$ & $0.13^{*}$ & 0.08 \\
\hline
\end{tabular}

Figures represent standardised regression coefficients for the longitudinal relation between assessments of arthritis and CTX-II. The higher the value of the standardised regression coefficient, the stronger is the longitudinal relation between indices of arthritis and CTX-II. Asterisks indicate the level of significance for testing the hypothesis that the standardised regression coefficient $=0$ (no relation): ${ }^{*} p<0.05,{ }^{* *} p<0.01$, ${ }^{* * *} p<0.001$.

Model with three months time lag:

$\operatorname{lnCTX}-I_{t}=$ constant $+a_{1} \cdot \operatorname{RF}(0,1)+a_{2} \cdot \operatorname{treatment}(0,1)+a_{3} \cdot \operatorname{RD}(0,1)+$ $b_{1} \cdot \mathrm{ESR}_{\mathrm{t}-3 \text { months }}+b_{2} \cdot \ln \mathrm{CTX}-\mathrm{II}_{\mathrm{t}-1}+c_{1} \cdot \mathrm{t}+\mathrm{c}_{2} \cdot \mathrm{t}^{2}$

Model with six months time lag:

$\ln \mathrm{CTX}-\mathrm{II}_{\dagger}=$ constant $+a_{1} \cdot \operatorname{RF}(0,1)+a_{2} \cdot$ treatment $(0,1)+a_{3} \cdot \operatorname{RD}(0,1)+$ $b_{1} \cdot \mathrm{ESR}_{\mathrm{t}-6 \text { months }}+b_{2} \cdot \ln \mathrm{CTX}-\|_{\mathrm{t}-1}+c_{1} \cdot \mathrm{t}+c_{2} \cdot \mathrm{t}^{2}$

Small letters in italics are regression coefficients: a refers to baseline variables (fixed); $b$ refers to longitudinal variables (time dependent), and $c$ refers to time variables $(R F$, rheumatoid factor $(0=$ absent; $1=$ present); treatment $(0=$ sulfasalazine; $1=C O B R A) ; R D$, radiographic damage at baseline ( 0 =absent; 1 = present); InCTX-II, the natural logarithm of CTXII. See text for further explanations).

CTX-II, collagen type II; DAS28, 28 joint disease activity score; ESR,

erythrocyte sedimentation rate. considered likely on the basis of the correlation between arthritis (expressed either as time averaged disease activity, or per separate joint) and radiographic progression, but was never proved in a one to one fashion.

The longitudinal type of analysis is crucial here, because, in contrast to cross sectional types of regression analysis, it introduces temporality into the association between arthritis and collagen breakdown. The autoregressive regression, in which every individual's value of CTX is adjusted for the previous individual's value, introduces an element of change and allows an interpretation of change (a higher level of ESR in an individual is associated with an increase in CTX, irrespective of the previous level); the time lag analysis unequivocally demonstrates that markers of the extent of arthritis are optimally associated with CTX levels if assessed in close temporal relation to CTX assessment, whereas the relation becomes weaker with increasing time lag; and finally, generalised estimating equations-the technique used here to quantify the relation between the extent of arthritis and CTX-ensures that within-patient correlation (a phenomenon that may be responsible for spurious relations in longitudinal studies) is properly adjusted for.

The results of our study suggest a better relation between arthritis and CTX-II than between arthritis and CTX-I. CTX-II reflects collagen type II degradation. Collagen type II degradation refers to cartilage degradation. The only type of cartilage that degrades in rheumatoid arthritis is the cartilage in joints. The assay used to measure CTX-II is highly specific, as argued previously, and CTX-II has been shown by us to be associated with long term radiographic progression. ${ }^{5}$ Thus, although this is not formally proven, it is very likely that the CTX-II measured in rheumatoid arthritis reflects cartilage breakdown.

CTX-I measures collagen type I degradation. Unlike CTXII, CTX-I reflects generalised as well as localised bone loss in rheumatoid arthritis. Generalised bone loss occurs in patients with rheumatoid arthritis for various reasons and it may easily obscure the subtle changes in CTX-I that can result from the degradation of localised bone (joints). This could explain the lower grade of association between signs of arthritis and CTX-I.

The results of our study do not exclude the possibility that part of the damage occurring in rheumatoid arthritis is independent of clinically perceptible arthritis. They show that arthritis causes collagen type I and type II degradation, that the extent of arthritis determines the extent of collagen degradation, and that it is very likely that clinically 
perceptible arthritis is detrimental to the joints in terms of structural damage. However, there is a possibility that progression of damage continues in particular patients who are free of arthritis, or alternatively, that progression of damage halts while arthritis is still clinically visible, as was suggested in clinical trials with tumour necrosis factor blocking drugs.

What are the clinical implications of the findings presented here? Assuming that the biomarkers CTX-I and CTX-II indeed reflect forthcoming structural damage, the close relation shown here between arthritis and CTX emphasises the need to suppress arthritis by means of drugs as soon and as thorough as possible, in order to limit future structural damage. In turn, the level of collagen type I and type II degradation, as measured by these biomarkers, may be used as a benchmark for the success of antirheumatic therapy with respect to the long term outcome. We already showed that antirheumatic therapy can immediately reduce CTX-II levels, and that the level of reduction is predictive of five year radiographic progression. ${ }^{6}$ This underscores the rationale for such a benchmark strategy.

\section{Authors' affiliations}

R B M Landewé, P Geusens, D M F M van der Heijde, S van der Linden, Department of Internal Medicine/Rheumatology, University Hospital Maastricht, Maastricht, Netherlands M Boers, Department of Clinical Epidemiology and Biostatistics, Free University Hospital, Amsterdam, Netherlands

P Garnero, INSERM and Synarc, Lyon, France

\section{REFERENCES}

1 Plant MJ, Williams AL, O'Sullivan MM, Lewis PA, Coles EC, Jessop JD. Relationship between time-integrated $C$-reactive protein levels and radiologic progression in patients with rheumatoid arthritis. Arthritis Rheum 2000;43:1473-7.

2 van Leeuwen MA, van Rijswijk MH, van der Heijde DM, Te Meerman GJ, van Riel PL, Houtman PM, et al. The acute-phase response in relation to radiographic progression in early rheumatoid arthritis: a prospective study during the first three years of the disease. Br J Rheumatol 1993;32(suppl 3):9-13.

3 van Leeuwen $M A$, van Rijswijk MH, Sluiter WJ, van Riel PL, Kuper IH, van der Putte $\mathrm{LB}$, et al. Individual relationship between progression of radiological damage and the acute phase response in early rheumatoid arthritis. Towards development of a decision support system. J Rheumatol 1997;24:20-7.

4 Welsing $P$, Landewe RB, van Riel $P$, Boers $M$, van Gestel $A M$, van der Linden S, et al. The relationship between disease activity and radiological progression in rheumatoid arthritis: A longitudinal analysis. Arthritis Rheum 2004;50:2082-93.

5 Garnero P, Landewe R, Boers M, Verhoeven A, van der Linden S, Christgau S, et al. Association of baseline levels of markers of bone and cartilage degradation with long-term progression of joint damage in patients with early rheumatoid arthritis: the COBRA study. Arthritis Rheum 2002;46:2847-56.

6 Landewe R, Geusens P, Boers M, van der Heijde D, Lems W, et al. Markers for type II collagen breakdown predict the effect of disease-modifying treatment on long-term radiographic progression in patients with rheumatoid arthritis. Arthritis Rheum 2004;50:1390-9.

7 Christgau S, Garnero P, Fledelius C, Moniz C, Ensig M, Gineyts E, et al. Collagen type II C-telopeptide fragments as an index of cartilage degradation. Bone 2001;29:209-15.

8 Boers M, Verhoeven AC, Markusse HM, van de Laar MA, Westhovens R, van Denderen JC, et al. Randomised comparison of combined step-down prednisolone, methotrexate and sulphasalazine with sulphasalazine alone in early rheumatoid arthritis. Lancet 1997;350:309-18.

9 Landewe RB, Boers M, Verhoeven AC, Westhovens R, van de Laar MA, Markusse $\mathrm{HM}$, et al. COBRA combination therapy in patients with early rheumatoid arthritis: long-term structural benefits of a brief intervention. Arthritis Rheum 2002;46:347-56.

10 Zeger SL, Liang KY. Longitudinal data analysis for discrete and continuous outcomes. Biometrics 1986;42:121-30. 